\title{
Physical Description of Franklin's Manuscript
}

PAPER

Endpapers watermarked: L Ruchaud / H V

Flyleaves not watermarked.

Pp. I-87, Pro Patria watermark with countermark H F. The leaf after p. 72 watermarked Arms of Amsterdam.

Pp. 88-I I I, watermark is not clear but has a central design of three five-pointed stars arranged horizontally, countermark is two lines of inscription surmounted by a crowned letter T (possibly J. Nourisson of Thiers, I73I, as the letters ". . . SSON FIN" are distinguishable). The paper has a bluish tinge.

Pp. I I 2-I 57, watermarked Fleur-de-lis and countermark G H.

Pp. 158-16I, watermarked Arms of Amsterdam, as in the leaf after p. 72, with counter mark I V (possibly J. Villedary, 1722 ).

Pp. 162-I66, watermarked a plow with countermark: J G \& Co / Brandywine.

Probably made at the Gilpin mills.

Pp. I67-1 82, same as pp. I I $2-157$.

Pp. 183-196*, same as pp. $15^{8-161 . ~}$

Pp. $197^{*}-202$, same as pp. I62-166.

Pp. 203-206, same as pp. I 58-16r.

Pp. 207-214, same as pp. I62-166.

Pp. 21 5-220, same as pp. 162-166, but the paper is whiter.

\section{HANDWRITING}

The manuscript is in the handwriting of Franklin, with the exception of the one leaf added after p. 72 and a marginal note on p. Igo written in ink by William Temple Franklin over a pencil inscription by Franklin. The page numeration throughout the manuscript is in two different hands.

Part I. Pp. I-87. Begun at Twyford, England, I77I.

Part II. Pp. 88-104. Written at Passy, France, in 1784 .

Part III. Pp. 104-213. Written in America, 1788-1789.

Part IV. Pp. $213^{-e n d . ~ W r i t t e n ~ i n ~ A m e r i c a, ~} 1789$ or 1790.

*After p. 199 the numbers revert to 190 in error; this reference is to the second series.

$$
\text { [xi] }
$$




\section{PAGINATION}

I-87. Every page is numbered. After p. 72 one leaf has been inserted on which is copied the text of the ink-stained page. This inserted leaf is not included in the regular sequence of numbers. The page following p. 87 is blank and not numbered.

88-2I9. These numbers are written in a larger, firmer hand and blacker ink. Paginated, but the numerals are written usually on the recto of the leaf, rarely also on the verso. From p. 88 onward the numerals were written before binding, as was probably true of the first 87 pages also. Page 167 is blank and not included in the numeration. After p. I99 the numbers revert to I90 in error. " $22 \mathrm{I}$ " is written upside down on the stub of the last leaf in the same hand as the other numbers.

Franklin numbered the sheets, i.e., each gathering of two conjugate leaves, at the top from I (p. I) to 2 I (p. 8I). The following 22 and 23 are single leaves only. Starting afresh with I (p. 88) written at the bottom, he continued to I7 (p. I $5^{2}$ ), then inserted A (p. I 56), B (p. I 58), C (p. I60), each of these last three a separate leaf, D (p. 162, a sheet again), E (p. I66, a leaf), and continued with 18 (p. I67) to 28 (p. I97*), the last so numbered by Franklin.

\section{BINDING}

Red pasteboard covers, red morocco spine, green vellum corners. Size: $133 / 4$ by $93 / 8$ inches. Labeled on the spine: THE LIFE / OF / FRANCKLIN. Ca. I790 to I8IO. Evidently French work.

\footnotetext{
* See preceding note.
} 